\title{
Resonant Excitation of Volume and Surface Fields on Complex Electrodynamic Surfaces
}

\author{
A.J. MacLachlan, ${ }^{1}$ C.W. Robertson, ${ }^{1}$ I.V. Konoplev, ${ }^{2}$ A.W. Cross,${ }^{1}$ A.D.R. Phelps, ${ }^{1,}{ }^{*}$ and \\ K. Ronald ${ }^{1}$ \\ ${ }^{1}$ Department of Physics, SUPA, University of Strathclyde, Glasgow G4 ONG, UK \\ ${ }^{2}$ John Adams Institute, Department of Physics, University of Oxford, Oxford OXI 3RH, UK
}

(Received 27 April 2018; revised manuscript received 26 January 2019; published 14 March 2019)

\begin{abstract}
Analytical, numerical, and experimental studies of volume and surface-field coupling in planar metal periodic surface lattice (PSL) structures superimposed on dielectric substrates with a metallic backing (PSLDM) are presented. We show the formation of frequency-locked PSLDM-coupled eigenmodes and unlocked surface-field resonances (PSL without substrate). These experimental observations are in excellent agreement with theoretical and numerical predictions. For the first time, the derivation of a field coupling coefficient $\alpha$ is demonstrated. By comparing theoretical and numerical dispersions, we obtain $\alpha$. Detailed analysis of possible scattering mechanisms and dispersive behavior in subwavelength "effective metadielectric" PSLs is shown. The theory and measurements presented in this paper are applicable over a broad frequency range from optical frequencies to $\mathrm{THz}$ and are fundamental to the innovation of high-power short-wavelength sources, solar cells, and alternative subwavelength absorbers.
\end{abstract}

DOI: 10.1103/PhysRevApplied.11.034034

\section{INTRODUCTION}

Advances in nanofabrication have stimulated renewed widespread interest in artificial surface materials that can exhibit unique electromagnetic (em) characteristics not otherwise observed in nature and dependent on their specific subwavelength structure. Unusual and intriguing phenomena, for instance, metamaterials with a high refractive index [1], negative permittivity [2-4], negative permeability [5-7], negative phase and group velocity at optical frequencies [8], and artificial magnetism [7,9-11] have been reported, leading to the realization of alternative and exciting applications including perfect lensing [12] and em cloaking [13]. Coupling of em fields in Periodic Surface Lattice (PSL) structures is another desirable property currently under intensive investigation [14-17]. Despite having a shallow subwavelength corrugation, these PSL structures do not satisfy the strict definition of a metadieletric. Instead, we consider such PSLs as high-impedance surfaces (with a finite depth) or "effective metadielectrics."

One inherent feature of the PSL structures is their capacity to support both volume and surface fields. Under

*a.d.r.phelps@strath.ac.uk

Published by the American Physical Society under the terms of the Creative Commons Attribution 4.0 International license. Further distribution of this work must maintain attribution to the author(s) and the published article's title, journal citation, and DOI. optimum conditions, PSLs facilitate the coupling of volume and surface fields to produce a single high $Q$ eigenmode. Interaction of this "effective metadielectric" eigenmode, for example, with an appropriate electron beam, is fundamental to the innovation of high-power radiation sources [14-19]. Unlike conventional microwave sources where the interaction region's size is limited by the operating wavelength, the proposed mode-coupling method supports single-mode operation in a large PSL cavity promising enhanced output power capabilities that can be exploited within a number of applications including perfect field absorbers [20], imaging [21-23], communications [24] astronomy, spectroscopy [25,26], particle beam diagnostics [27], particle acceleration [28], and solar cells [29]. The concepts we develop in this paper are independent of frequency and are relevant to many branches of physics that utilize em-field coupling [30] and wave-matter interactions. The "proof-of-principle" coupling is applicable to devices incorporating different types of gain media, including semiconductor materials, typically used in condensed matter physics.

For cylindrical metallic PSLs compatible with an electron beam, the scatterers are synchronized by a near cut-off $\mathrm{TM}_{0, T}$ volume mode $[14,15]$. The fundamental "proof-of-principle" coupling of volume and surface modes is demonstrated using PSLs with simpler planar geometry mounted on copper-backed substrates. The structures are based on printed circuit boards (PCBs) composed of a 35- $\mu \mathrm{m}$-copper coating on both sides of a commercial 


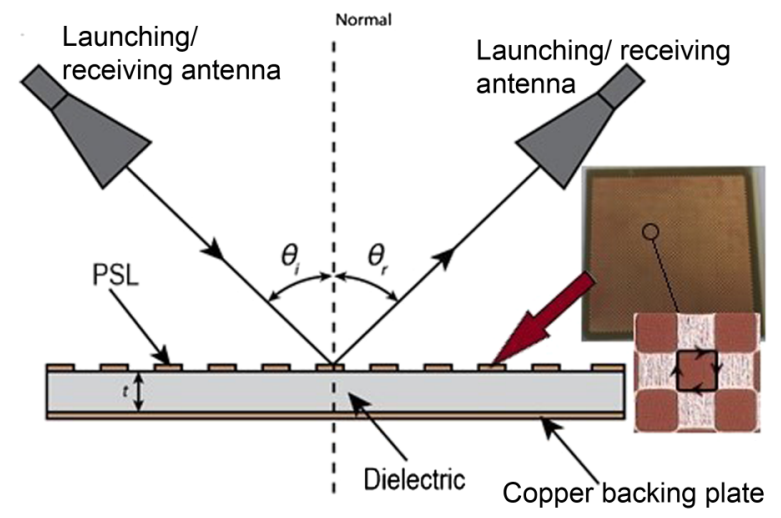

FIG. 1. Schematic showing the planar PSLDM structure. The PSL acts as a partially transparent mirror 1 and is mounted on a dielectric substrate of thickness $t$ with a copper backing that forms mirror 2, similar to a Fabry-Perot cavity. The inset photograph (and magnified image) show a planar PSL (period, $1.94 \mathrm{~mm}$ ) etched onto a copper-backed substrate of thickness $0.76 \mathrm{~mm}$. The directional arrows indicate the induced surface current flowing around each lattice cell. The surface currents are scattered into propagating (volume) waves that form the eigenmode of the structure.

fiberglass-epoxy (FR-4) composite with relative permittivity $\varepsilon_{r}=4.3$. Planar PSLs are etched into the top copper layer using a chemical etchant to remove the copper coating in defined regions exposing the FR-4 substrate to form the lattice cells, as photographed in the inset of Fig. 1. Instead of using a cut-off volume mode as in the cylindrical geometry, lattice synchronization is delivered via a Fabry-Perot configuration with the PSL and copper-backing plate in place of mirrors (Fig. 1). Clearly, the conversion between the PSLs of planar (not intended for deployment within an electron beam) and cylindrical geometries is possible using conformal mapping. For the first time, the detailed derivation of a coupling coefficient $\alpha$, which governs the eigenmode's dispersive properties and is required in applications, is presented. Evaluation of $\alpha$ involves carrying out a complex integral mode coupling calculation and a good estimation of $\alpha$ is obtained by comparing the theoretical (developed for a cylindrical PSL and also applicable to a planar PSL in the case of a large radius) and numerical models. Agreement between theory, modeling, and experimental measurements is reported in this paper.

\section{THEORY}

The PSL's eigenfield for the case of an oversized cylindrical PSL can be described as a superposition of a welldefined $\mathrm{TM}_{0, T}$ volume field and high-order $\mathrm{TM}_{\bar{m}, n}$ surface fields. This theory is also applicable to planar PSLs based on the assumption that the mean radius of the cylinder $r_{0}$ is large in comparison to the operating wavelength $\left(r_{0} \gg \lambda\right)$, which is made throughout this analysis. Lattice synchronization is obtained by launching a near-cut-off symmetric $\mathrm{TM}_{0, T}$ volume mode (where $T$ and 0 are the numbers of radial and azimuthal variations, respectively) through the structure, or in the case of planar geometry, by exciting a Fabry-Perot standing plane wave that exists between mirrors 1 and 2 (Fig. 1) due to scattering of the surface currents. The cylindrical PSL can be substituted for a smooth waveguide with a fictitious magnetic surface current density $\mathbf{j}_{m}$ (symbols in bold font indicate 3 -vectors) in place of the corrugation to describe field coupling or scattering. Assuming $r_{0}$ is large, the theory developed for a cylindrical PSL is also relevant to the study of an equivalent planar PSL.

From Maxwell's curl equations, $\nabla^{2} \mathbf{H}=-\omega^{2} \varepsilon \mu \mathbf{H}+$ $i \omega \varepsilon \mathbf{j}_{m}$ where $\varepsilon=\varepsilon_{r} \varepsilon_{0}$ and $\mu=\mu_{r} \mu_{0}$ denote, respectively, the permittivity and permeability in the "effective metadielectric" material. In the theoretical study, we take $\varepsilon_{r}=1$ and $\mu_{r}=1$ in the case of vacuum. When $r_{0} \gg \lambda_{\text {op }}$ and providing the corrugation $\Delta r$ is suitably shallow, $\left(\Delta r \ll \lambda_{\text {op }}\right)$, the PSL's eigenfield can be described as a superposition of waveguide modes (where the volume and surface modes have real and imaginary transverse wavenumbers, respectively). The transverse electric $\mathbf{E}$ and magnetic $\mathbf{H}$ fields are expanded as a sum of the waveguide modes $\mathbf{H}, \mathbf{E}=$ $\sum_{q}\left[C_{q}(z), D_{q}(z)\right]\left(\mathbf{H}_{q}, \mathbf{E}_{q}\right)$, allowing the field inside the finite cylinder to be described as a slow wave envelope filled with fast varying terms. The field inside the structure is decomposed as a set of eigenfields, where $C_{q}(z)$ and $D_{q}(z)$ are the slowly varying magnetic and electric eigenfield amplitudes, respectively.

$$
\begin{array}{r}
\sum_{q} \nabla^{2}\left[C_{q}(z) \mathbf{H}_{q}\right]=-\omega^{2} \varepsilon \mu \sum_{q} C_{q}(z) \mathbf{H}_{q}+i \omega \varepsilon \mathbf{j}_{m}, \\
\sum_{q} \nabla^{2}\left[D_{q}(z) \mathbf{E}_{q}\right]=-\omega^{2} \varepsilon \mu \sum_{q} D_{q}(z) \mathbf{E}_{q} .
\end{array}
$$

Equations (1a) and (1b) describe the structure's fields in their general form and volume and surface field amplitudes are introduced later in the theory. To succinctly describe the field distribution inside, it is sufficient to define only the magnetic fields as the electric fields can be derived from them. The total power transmitted through the structure is defined by integrating the Poynting vector $(1 / 2)\left(\mathbf{E} \times \mathbf{H}^{*}\right)$ over the cylindrical cross section. This treatment is restricted to consider only the near cut-off volume mode $\left(\omega \approx \omega_{0}^{v}\right)$ for which $k_{z} \rightarrow 0$ and $\lambda_{z} \rightarrow \infty$. In the case of a planar system, the structure is assumed to be infinitely large and edge effects are neglected. In practice, however, some detuning from the ideal situation where $\omega=\omega_{0}$ exists due to the structure's finite length. $\mathbf{E} \times$ $\mathbf{H}^{*}$ is implicitly defined by multiplying both sides of Eq. (1a) by the complex conjugate of the transverse magnetic cut-off mode $\left(\mathbf{H}_{q^{\prime}}^{*}\right)$. Separating $\nabla^{2}$ into its transverse and 
longitudinal components and substituting the Helmholtz equation $\nabla_{\perp}^{2} \mathbf{H}_{q}+\left(\left(\omega_{0}^{q}\right)^{2} / c^{2}\right) \mathbf{H}_{q}=0$ for the localized volume and surface fields into $\mathbf{H}_{q^{\prime}}^{*} \cdot \sum_{q} \nabla_{z}^{2} C_{q}(z) \mathbf{H}_{q}+\mathbf{H}_{q^{\prime}}^{*}$. $\sum_{q} \nabla_{\perp}^{2} C_{q}(z) \mathbf{H}_{q}+(\omega / c)^{2} \quad \mathbf{H}_{q^{\prime}}^{*} \cdot \sum_{q} C_{q}(z) \mathbf{H}_{q}=i \omega \varepsilon \mathbf{j}_{m} \cdot \mathbf{H}_{q^{\prime}}^{*}$ gives

$$
\begin{gathered}
\mathbf{H}_{q^{\prime}}^{*} \cdot \sum_{q} \nabla_{z}^{2} C_{q}(z) \mathbf{H}_{q}+\mathbf{H}_{q^{\prime}}^{*} \cdot \sum_{q} C_{q}(z)\left(\frac{\omega^{2}-\omega_{0}^{2}}{c^{2}}\right) \\
\mathbf{H}_{q}=i \omega \varepsilon \mathbf{j}_{m} \cdot \mathbf{H}_{q^{\prime}}^{*},
\end{gathered}
$$

where $q^{\prime}$ denotes the near cut-off volume mode. Imposing the orthogonality condition $\int_{S_{\perp}} \mathbf{H}_{q} \cdot \mathbf{H}_{q^{\prime}}^{*} d \sigma=0$ if $q \neq q^{\prime}$ and integrating over the waveguide's cylindrical crosssection $S_{\perp}$, where $d \sigma$ is the differential element of the surface area, gives the normalized wave equation. Small diffractive and Ohmic losses are considered by introducing detuning parameters. Here, we introduce detuning between the variable frequency $\omega$ and the system eigenfrequencies $\omega_{0}$ and $\omega_{0}^{v, s}$ (where $\omega_{0}$ is the cut-off frequency if no corrugation is introduced, i.e., pure volume wave as an ideal solution of Helmholtz equation, while $\omega_{0}^{v, s}$ are eigenfrequencies of the volume and surface fields, respectively, if corrugations are introduced) is $\left(\omega^{2}-\omega_{0}^{2}\right) / c^{2}$, while the detuning between volume and surface modes is $\Delta=\omega_{0}^{v}-\omega_{0}^{s} \cong c k_{z}^{v}$. We note that as corrugations are shallow, $\omega_{0} \cong \omega_{0}^{v}$, satisfying the Bragg resonance condition $\bar{k}_{z}=k_{z}^{s}-k_{z}^{v}$ for the near cut-off $\left(k_{z}^{v} \cong 0\right) \mathrm{TM}_{0, T}$ mode requires that $\omega_{B}=\omega_{0}^{s}$. We define the Bragg detuning $\hat{\delta}=$ $\left[2\left(\omega_{B}-\bar{\omega}_{0}\right) / c^{2}\right]$ in terms of the mean angular frequency, $\bar{\omega}_{0}=\left[\left(\omega_{0}^{v}+\omega_{0}^{s}\right) / 2\right]$ and incorporate $\hat{\delta}$ into the normalized wave equation. We note that $\hat{\delta}$ can be complex and its imaginary part describes the losses.

$$
\nabla_{z}^{2} C_{q}^{v, s}(z)+\omega \hat{\delta} C_{q}^{v, s}(z) \mp \frac{\bar{\omega}_{0} \Delta}{c^{2}} C_{q}^{v, s}(z)=N_{v, s} \oint \mathbf{j}_{m} \cdot \mathbf{H}_{q^{\prime}}^{*} d \sigma
$$

$N_{v, s}=\left(i \omega \varepsilon / \oint_{S_{\perp}} \mathbf{H}_{q} \cdot \mathbf{H}_{q^{\prime}}^{*} d \sigma\right)$ is the wave norm for the volume $(v)$ and surface $(s)$ modes, for which the "-" and "+" signs apply, respectively. The integral $\oint_{S_{\perp}} \mathbf{H}_{q} \cdot \mathbf{H}_{q^{\prime}}^{*} d \sigma$ denotes a closed surface integration with the boundaries defined by the smooth boundaries of the unperturbed waveguide.

Due to the structure's periodicity, it is possible to express the slowly varying surface field as a complex Fourier series of the form $C_{q}^{s}(z)=\sum_{n_{s}=-\infty}^{\infty} B_{n_{s}}(z) e^{i n_{s} \bar{k}_{z} z}$ where $B_{n_{s}}$ is the amplitude of the surface field's $n_{s}$ harmonic. Likewise, the volume field can be written as a complex Fourier expansion using the approximation $k_{z, v} \cong$ $\bar{k}_{z}=2 \pi / d_{z}$, which equates the volume field's longitudinal wavenumber to that of the structure and corresponds to the case of coherent coupled eigenmode formation, that is, minimal detuning. Evaluating the second-order partial differential terms and substituting into Eq. (3) yields wave equations for the volume Eq. (4a) and surface Eq. (4b) modes.

$$
\begin{aligned}
& \sum_{n_{v}} {\left[e^{i n_{v} \bar{k}_{z} z} \frac{\partial^{2} A_{n_{v}}(z)}{\partial z^{2}}+2 i n_{v} \bar{k}_{z} e^{i n_{v} \bar{k}_{z} z} \frac{\partial A_{n_{v}}(z)}{\partial z}\right.} \\
&-\left.n_{v}^{2} \bar{k}_{z}^{2} A_{n_{v}}(z) e^{i n_{v} \bar{k}_{z} z}\right]+\omega \hat{\delta}\left[\sum_{n_{v}} A_{n_{v}}(z) e^{i n_{v} \bar{k}_{z} z}\right] \\
&-\frac{\bar{\omega}_{0} \Delta}{c^{2}}\left[\sum_{n_{v}} A_{n_{v}}(z) e^{i n_{v} \bar{k}_{z} z}\right]=N_{v} \oint \mathbf{j}_{m} \cdot \mathbf{H}_{q^{\prime}}^{*} d \sigma, \\
& \sum_{n_{S}}\left[e^{i n_{s} \bar{k}_{z} z} \frac{\partial^{2} B_{n_{s}}(z)}{\partial z^{2}}+2 i n_{s} \bar{k}_{z} e^{i n_{s} \bar{k}_{z} z} \frac{\partial B_{n_{s}}(z)}{\partial z}\right. \\
&\left.-n_{s}^{2} \bar{k}_{z}^{2} B_{n_{s}}(z) e^{i n_{s} \bar{k}_{z} z}\right]+\omega \hat{\delta}\left[\sum_{n_{s}} B_{n_{s}}(z) e^{i n_{s} \bar{k}_{z} z}\right] \\
&-\frac{\bar{\omega}_{0} \Delta}{c^{2}}\left[\sum_{n_{s}} B_{n_{s}}(z) e^{i n_{s} \bar{k}_{z} z}\right]=N_{s} \oint \mathbf{j}_{m} \cdot \mathbf{H}_{q^{\prime}}^{*} d \sigma,
\end{aligned}
$$

where $A_{n_{v}}$ is the amplitude of the $n_{v}$ harmonic of the volume field. To evaluate the righthand sides of Eqs. (4a) and (4b), we must define $\mathbf{j}_{m} \cdot \mathbf{H}_{q}^{*}$. A cylindrical PSL with sinusoidal corrugation $l=r_{0}+\Delta r \cos (\bar{m} \varphi) \cos \left(\bar{k}_{z} z\right)$ and $\bar{m}$ azimuthal variations $[14,15]$ is equivalent to a smooth cylindrical waveguide when the magnetic surface current boundary condition $\mathbf{j}_{m}=\mathbf{n} \times[\nabla(l \mathbf{E} \cdot \mathbf{n})]+i \omega l \mathbf{n} \times$ $[\mathbf{n} \times \mathbf{H}]$ is satisfied [31]. For planar geometry, $\bar{m} \varphi$ is substituted with $\bar{k}_{y} y$ where $\bar{k}_{y}=2 \pi / d_{y}$. Enforcing this condition gives $\mathbf{j}_{m} \cdot \mathbf{H}_{q}^{*}=i \omega l(z, \varphi)\left(\mathbf{E}_{q, n} \cdot \mathbf{E}_{q, n}^{*}+\mathbf{H}_{q, \tau} \cdot \mathbf{H}_{q, \tau}^{*}\right)$, where $\mathbf{E}_{q, n}$ and $\mathbf{H}_{q, \tau}$ are the normal electric and tangential magnetic field components with complex conjugates $\mathbf{E}_{q, n}^{*}$ and $\mathbf{H}_{q, \tau}^{*}$. In the case of a $T M_{0, T}$ volume mode (with no normal electric field component), $\mathbf{E}_{q, n}=0$. Separating $\mathbf{H}_{q, \tau}$ into its volume and surface field constituents $\mathbf{H}_{q, \tau}=$ $\mathbf{H}_{q, \tau}^{v}(r) C_{q}^{v}(z)+\mathbf{H}_{q, \tau}^{s}(r, \varphi) C_{q}^{s}(z)$ and expanding to include the full set of eigenmodes gives

$$
\begin{array}{r}
\mathbf{j}_{m} \cdot \mathbf{H}_{q}^{*}=i \omega\left[r_{0}+\frac{\Delta r}{4}\left(e^{i \bar{m} \varphi}+e^{-i \bar{m} \varphi}\right)\left(e^{i \bar{k}_{z} z}+e^{-i \bar{k}_{z} z}\right)\right] \mathbf{H}_{q, \tau}^{*, v} . \\
{\left[\mathbf{H}_{q, \tau}^{v}(r) \sum_{2} C_{q}^{v}(z)+\mathbf{H}_{q, \tau}^{s}(r) \cos \left(m_{s} \varphi\right) \sum_{n_{s}} B_{n_{s}}(z) e^{i n_{s} \bar{k}_{z} z}\right] .}
\end{array}
$$

The $\mathbf{j}_{m} \cdot \mathbf{H}_{q}^{*}$ term is now integrated over the cylindrical cross section $d \sigma$, where $N_{v, s}^{\prime} \oint \mathbf{j}_{m} \cdot \mathbf{H}_{q, \tau}^{*(v, s)} d \sigma=$ $\left.N_{v, s}^{\prime} \int_{0}^{2 \pi} r\left(\mathbf{j}_{m} \cdot \mathbf{H}_{q}^{*}\right)\right|_{r=r_{0}} d \varphi$ and $N_{v, s}^{\prime}=i \omega N_{v, s}$. One of the 
necessary criteria for volume and surface mode coupling is $\oint \mathbf{j}_{m} \cdot \mathbf{H}_{q}^{*} d \sigma \neq 0$. This requirement is satisfied by averaging over the period of fast oscillations from 0 to $2 \pi$, neglecting exponential (oscillating) terms that would otherwise integrate to zero. The fundamental volume field harmonic $\left(n_{v}=0\right)$ is first described in the form $\oint \mathbf{j}_{m} \cdot \mathbf{H}_{q}^{*} d \sigma=\left.r_{0}^{2} N_{v}^{\prime} \int_{0}^{2 \pi}(I)\right|_{r=r_{0}} d \varphi$, where $I$ is the integrand, composed of four terms

(1) $\mathbf{H}_{q, \tau}^{* v}(r) \cdot \mathbf{H}_{q, \tau}^{v}(r) \sum_{2} C_{q}^{v}(z)$,

(2) $\mathbf{H}_{q, \tau}^{* v} \cdot \mathbf{H}_{q, \tau}^{s}(r) \cos \left(m_{s} \varphi\right) \sum_{n_{s}} B_{n_{s}}(z) e^{i n_{s} \bar{k}_{z} z}$,

(3) $\frac{\Delta r}{4 r_{0}}\left(e^{i \bar{m} \varphi}+e^{-i \bar{m} \varphi}\right)\left(e^{i \bar{k}_{z} z}+e^{-i \bar{k}_{z} z}\right)$

$\times \mathbf{H}_{q, \tau}^{* v} \cdot \mathbf{H}_{q, \tau}^{v}(r) \sum_{2} C_{q}^{v}(z)$,

(4) $\frac{\Delta r}{4 r_{0}}\left(e^{i \bar{m} \varphi}+e^{-i \bar{m} \varphi}\right)\left(e^{i \bar{k}_{z} z}+e^{-i \bar{k}_{z} z}\right) \mathbf{H}_{q, \tau}^{* v}$.

$$
\mathbf{H}_{q, \tau}^{s}(r) \cos \left(m_{s} \varphi\right) \sum_{n_{s}} B_{n_{s}}(z) e^{i n_{s} \bar{k}_{z} z} .
$$

Terms 2 and 4 demonstrate scattering and potential coupling of volume and surface fields. The geometric parameter $\Delta r / r_{0}$ is closely linked to the coupling coefficient. Discarding terms 2 and 3 (which integrate to zero after averaging over the fast oscillation terms) and employing the trigonometric identity $\cos \left(m_{s} \varphi\right)=1 / 2\left(e^{i m_{s} \varphi}+e^{-i m_{s} \varphi}\right)$ gives

$$
\begin{gathered}
\oint \boldsymbol{j}_{m} \cdot \mathbf{H}_{q}^{*} d \sigma=r_{0}^{2} N^{\prime}{ }_{v} \int_{0}^{2 \pi}\left[\mathbf{H}_{q, \tau}^{* v}\left(r_{0}\right) \cdot \mathbf{H}_{q, \tau}^{v}\left(r_{0}\right) \sum_{2} C_{q}^{v}(z)\right. \\
+\frac{\Delta r}{8 r_{0}} \mathbf{H}_{q, \tau}^{* v}\left(r_{0}\right) \cdot \mathbf{H}_{q, \tau}^{s}\left(r_{0}\right)\left(e^{i \bar{k}_{z} z}+e^{-i \bar{k}_{z} z}\right)\left(e^{i \bar{m} \varphi}+e^{-i \bar{m} \varphi}\right) \\
\left.\left(e^{i m_{s} \varphi}+e^{-i m_{s} \varphi}\right) \sum_{n_{s}} B_{n_{s}}(z) e^{i n_{s} \bar{k}_{z} z}\right]\left.\right|_{r=r_{0}} d \varphi
\end{gathered}
$$

A nontrivial result is obtained only when $m_{s}=\bar{m}$, forcing the fast oscillation terms to vanish. Based on the azimuthal Bragg condition, $\bar{m}=m_{v}+m_{s}$, we establish that $m_{v}=0$, justifying the role of the azimuthally symmetric $T M_{0, T}$ volume mode. The remaining fast oscillation terms are eliminated when $n_{s}= \pm 1$. Until now, only the fundamental harmonic of the volume field has been considered. For completeness, and to provide a more thorough mathematical description of the possible scattering processes, the Fourier expansion of both fields is included, allowing different low-order values of $n_{v}$ and $n_{s}$ to be explored. We follow a similar procedure to that above, now multiplying $\mathbf{j}_{m} \cdot \mathbf{H}_{q}^{*}$ by $e^{-i \bar{k}_{z} z n_{v, s}}$ to describe possible coupling mechanisms involving the $n_{v, s}=0$ and $n_{v, s}= \pm 1,2$ field harmonics. The scattering of the $n_{v}=0$ volume field into the surface field is described by the expression

$$
\begin{gathered}
\pi r_{0}^{2} N_{v}^{\prime}\left[\mathbf{H}_{q, \tau}^{*, v}\left(r_{0}\right) \cdot \mathbf{H}_{q, \tau}^{v}\left(r_{0}\right) \sum_{n_{v}} A_{n_{v}}(z)+\frac{\Delta r}{2 r_{0}} \mathbf{H}_{q, \tau}^{*, v}\left(r_{0}\right) .\right. \\
\left.\mathbf{H}_{q, \tau}^{s}\left(r_{0}\right) \sum_{n_{s}} B_{n_{s}}(z)\left(e^{i \bar{k}_{z} z\left(1-n_{v}+n_{s}\right)}+e^{i \bar{k}_{z} z\left(-1-n_{v}+n_{s}\right)}\right)\right] .
\end{gathered}
$$

For a nonzero result after integrating, the conditions $1-$ $n_{v}+n_{s}=0 ; n_{s}^{1}=n_{v}-1$ and $-1-n_{v}+n_{s}=0 ; n_{s}^{2}=$ $n_{v}+1$ must hold true, demonstrating the potential for coupling of the fundamental volume field and $n_{s} \pm 1$ surfacefield harmonics. Likewise, scattering between the $n_{v} \pm 1$ and $n_{s}=0, \pm 2$ harmonics is also possible. Scattering of the surface field into the volume field is investigated in a similar manner, multiplying Eq. (5) by $e^{-i \vec{k}_{z} z n_{s}}$. Expanding and neglecting the same terms as before gives the following expression after integration

$$
\begin{gathered}
\pi r_{0}^{2} N_{s}^{\prime}\left[\mathbf{H}_{q, \tau}^{*, s}\left(r_{0}\right) \cdot \mathbf{H}_{q, \tau}^{s}\left(r_{0}\right) \sum_{n_{s}} B_{n_{s}}(z)+\frac{\Delta r}{2 r_{0}} \mathbf{H}_{q, \tau}^{*, s}\left(r_{0}\right) .\right. \\
\left.\mathbf{H}_{q, \tau}^{v}\left(r_{0}\right) \sum_{n_{v}} A_{n_{v}}(z)\left(e^{i \bar{k}_{z} z\left(1-n_{s}+n_{v}\right)}+e^{-i \bar{k}_{z} z\left(1+n_{s}-n_{v}\right)}\right)\right] .
\end{gathered}
$$

This expression is comprised of two parts, the first describing the accumulation of a localized surface field (which has a dissipative effect and does not contribute to the coupling of volume and surface fields) and the second defining scattering of the surface field into the volume field. Once again, to eliminate the exponential terms and obtain a nonzero result, the following conditions must be met: $1-n_{s}+n_{v}=0 ; n_{v}^{1}=n_{s}-1$ and $1+n_{s}-n_{v}=0 ; n_{v}^{2}=$ $n_{s}+1$. Coupling coefficients are introduced to describe the mutual resonant scattering (i.e., the volume field coupling with the surface field $\alpha_{v s}$ and the surface field coupling with the volume field $\alpha_{s v}$ ) leading to the following set of coupled wave equations.

$$
\begin{aligned}
& \nabla_{z}^{2} C_{q}^{v}(z)+\omega \hat{\delta} C_{q}^{v}(z)-\frac{\bar{\omega}_{0} \Delta}{c^{2}} C_{q}^{v}(z)=N_{v} \oint \mathbf{j}_{m} \cdot \mathbf{H}_{q^{\prime}}^{*} d \sigma \\
& \nabla_{z}^{2} C_{q}^{s}(z)+\omega \hat{\delta} C_{q}^{s}(z)+\frac{\bar{\omega}_{0} \Delta}{c^{2}} C_{q}^{s}(z)=N_{s} \oint \mathbf{j}_{m} \cdot \mathbf{H}_{q}^{*} d \sigma,
\end{aligned}
$$




$$
\begin{aligned}
& \frac{\partial^{2} A_{n}(z)}{\partial z^{2}}+2 i(n) \bar{k}_{z} \frac{\partial A_{n}(z)}{\partial z}-\bar{k}_{z}^{2}(n)^{2} A_{n}(z) \\
& \quad+\left(\omega \hat{\delta}-\frac{\bar{\omega}_{0} \Delta}{c^{2}}\right) A_{n}(z)=\alpha_{v s}\left[B_{n-1}(z)+B_{n+1}(z)\right]
\end{aligned}
$$

$$
\begin{aligned}
& \frac{\partial^{2} B_{n}(z)}{\partial z^{2}}+2 i(n) \bar{k}_{z} \frac{\partial B_{n}(z)}{\partial z}-\bar{k}_{z}^{2}(n)^{2} B_{n}(z) \\
& \quad+\left(\omega \hat{\delta}+\frac{\bar{\omega}_{0} \Delta}{c^{2}}\right) B_{n}(z)=\alpha_{s v}\left[A_{n-1}(z)+A_{n+1}(z)\right]
\end{aligned}
$$

Combining these coupling coefficients into a single parameter, we define $\alpha=\sqrt{\alpha_{v s} \alpha_{s v}}$. Finally, new amplitude constants, $\tilde{A}_{n_{v}}(z)=A_{n_{v}}(z) \sqrt{\alpha_{s v}} / \sqrt{\alpha_{v s}}$ and $\tilde{B}_{n_{s}}(z)=$ $B_{n_{s}}(z) \sqrt{\alpha_{v s}} / \sqrt{\alpha_{s v}}$ are introduced to give the following normalized coupled wave equations

$$
\begin{aligned}
& \frac{\partial^{2} \tilde{A}_{n}(z)}{\partial z^{2}}+2 i(n) \bar{k}_{z} \frac{\partial \tilde{A}_{n}(z)}{\partial z}-\bar{k}_{z}^{2}(n)^{2} \tilde{A}_{n}(z) \\
& \quad+\left(\omega \hat{\delta}-\frac{\bar{\omega}_{0} \Delta}{c^{2}}\right) \tilde{A}_{n}(z)=\alpha\left[B_{n-1}(z)+B_{n+1}(z)\right] \\
& \frac{\partial^{2} \tilde{B}_{n}(z)}{\partial z^{2}}+2 i(n) \bar{k}_{z} \frac{\partial \tilde{B}_{n}(z)}{\partial z}-\bar{k}_{z}^{2}(n)^{2} \tilde{B}_{n}(z) \\
& \quad+\left(\omega \hat{\delta}+\frac{\bar{\omega}_{0} \Delta}{c^{2}}\right) \tilde{B}_{n}(z)=\alpha\left[A_{n-1}(z)+A_{n+1}(z)\right]
\end{aligned}
$$

where the normalized coupling coefficient $\alpha$ is defined as

$$
\begin{aligned}
\alpha= & \frac{\pi r_{0} \Delta r}{2} \\
& \times \sqrt{N^{\prime}{ }_{v} N_{s}^{\prime}\left[\mathbf{H}_{q, \tau}^{*, v}\left(r_{0}\right) \cdot \mathbf{H}_{q, \tau}^{s}\left(r_{0}\right)\right]\left[\mathbf{H}_{q, \tau}^{*, s}\left(r_{0}\right) \cdot \mathbf{H}_{q, \tau}^{v}\left(r_{0}\right)\right]} .
\end{aligned}
$$

A coupled dispersion equation incorporating $\alpha$ and describing the structure's coupled eigenfield [15] is derived

$$
\begin{aligned}
& \left(\omega_{e}^{2}-\Lambda^{2}\right)\left[\Lambda^{4}-2 \Lambda^{2}\left(2+\Gamma^{2}+\omega_{e}^{2}\right)+\left(2-\Gamma^{2}+\omega_{e}^{2}\right)^{2}\right] \\
& \quad=2 \alpha^{4}\left(2-\Gamma^{2}+\omega_{e}^{2}-\Lambda^{2}\right),
\end{aligned}
$$

where $\Lambda$ is the normalized wave vector, $\omega_{e}=$ $\sqrt{\delta^{2}+2 \delta+\tilde{\Delta}^{2}}$ is a variable angular frequency, and $\delta=$ $(\omega-\Omega) / \Omega, \tilde{\Delta}=\Phi / \Omega$, and $\Gamma=2 \bar{k}_{z} c /\left[\left(\omega_{0}^{v}\right)^{2}+\left(\omega_{0}^{s}\right)^{2}\right]$ are renormalized detuning parameters with constants $\Phi, \Omega=\sqrt{\left[\left(\omega_{0}^{s}\right)^{2} \mp\left(\omega_{0}^{v}\right)^{2}\right] / 2}$. Solving Eq. (12) involves performing an integral mode calculation around the cylindrical cross section. Analytical dispersion diagrams can otherwise be obtained by treating $\alpha$ and $\Gamma$ as variable parameters [15]. Waveguide dimensions and Bessel roots were previously used to compute $\omega_{0}^{v}$ for the $T M_{0, T}$ volume field [15]. Here, we employ a different approach, instead comparing theoretical predictions with numerical modeling.

\section{SIMULATION RESULTS}

Numerical simulations are performed using Computer Simulation Technology (CST) Microwave Studio's (MWS) Advanced Krylov Subspace (AKS) Eigenmode Solver [32] to simulate a single lattice cell with periodic boundaries at the corrugation. Along the $y$ axis, boundary constraints on the tangential fields impose $H_{\tau}=0$ at the corrugated surface and $E_{\tau}=0$ at the metal wall. A parameter sweep is used to evaluate a specified number of eigenmodes over the phase range $-2 \pi$ to $2 \pi$. This solver assumes normal and uniform irradiation and does not calculate modes for a specified incident angle. A PSL with planar geometry (where the individual volume and surface fields that constitute the cavity eigenmode are unknown) is modeled and numerical dispersions are used to identify possible volume and surface field interactions. The CST MWS dispersion diagrams are compared to the equivalent (i.e., same $\omega_{0}^{v}, \Gamma$ ) dispersions based on the theoretical analysis. The planar and cylindrical geometries for which the two models are developed are related by the assumption that the mean radius of the cylindrical waveguide is very large $\left(r_{0} \gg \lambda\right)$. Differences between the two models, including the planar and cylindrical geometries of the PSL structures, are taken into account. For instance, just one specified volume mode is considered in the theoretical approach, whereas the CST MWS Eigenmode Solver computes a number of possible modes. Similarly, while the theoretical dispersion is solved for the fundamental volume mode, numerical calculations include the volume field's spatial harmonics. These coupled neighboring $n_{v}=$ \pm 1 harmonics modify the dispersion's gradient and overall appearance in the vicinity of $\theta=\pi$ (radians). Despite these differences, there are strong similarities between these dispersion diagrams.

Figure 2 shows dispersion diagrams for PSL structures with a periodicity of $1.94 \mathrm{~mm}$ and corrugation depth $\Delta r=35 \mu \mathrm{m}$ for (a) the planar PSLDM mounted on a 0.76-mm metal-backed FR-4 substrate (with relative permittivity $\epsilon_{r}=4.3$ ) modeled using CST MWS and (b) a cylindrical PSL with $r_{0} \gg \lambda$ solved using Eq. (12). In both instances, the volume field has $\omega_{0}^{v} \cong 286 \pi \times 10^{9}\left(\mathrm{rad} \mathrm{s}^{-1}\right)$ and $\alpha$ is treated as a variable parameter. The red traces ( 1 , 2) of Fig. 2(a) are coupled eigenmode dispersions and the maximum $(161 \mathrm{GHz})$ and minima $(150,168 \mathrm{GHz})$ frequencies of these branches indicate the positions of possible coupled-cavity eigenmodes. 


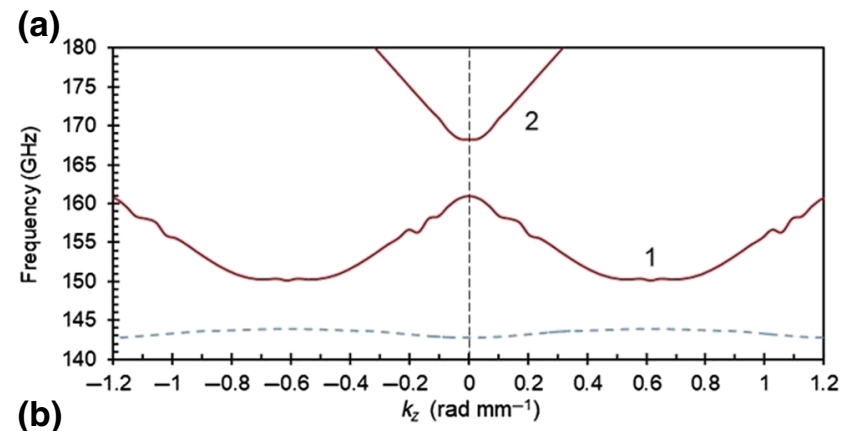

(b)

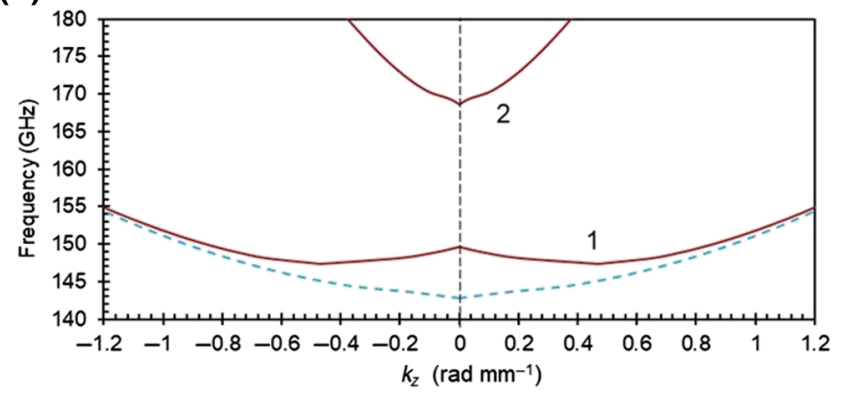

FIG. 2. Dispersion diagrams for PSL structures with a 1.94-mm periodicity. The solid red lines are coupled dispersion branches (formed from coupled volume and surface fields). The maxima and minima of these branches indicate the frequencies of coupled eigenmodes. (a) Dispersion of 1.94-mm planar PSL mounted on a copper-backed substrate and modeled using CST MWS Eigenmode solver (with $\Delta r=35 \mu \mathrm{m}, \varepsilon_{r}=4.3, t=0.76 \mathrm{~mm}$ ). The blue dashed line is the volume field coupled to its neighboring spatial harmonics. (b) Dispersion of the 1.94-mm oversized, cylindrical PSL (with $\omega_{0}^{v} \cong 286 \pi \times 10^{9} \mathrm{rad} \mathrm{s}^{-1}, \Gamma=1.44, \tilde{\Delta}=$ $0.33, \alpha=0.45$ ) obtained by solving Eq. (12). The blue dashed line is the fundamental volume field dispersion.

The volume field's dispersion (dashed line) is modified by its neighboring spatial harmonics. Each lattice cell resembles a rectangular waveguide aperture that supports a localized surface field when $\lambda_{s} \cong d_{z}$. The surface field's parameters are evaluated $\left(\omega_{0}^{s} \cong 310 \pi \times\right.$ $\left.10^{9}\left(\operatorname{rad~s}^{-1}\right) ; \Gamma=1.44 ; \tilde{\Delta}=0.33\right)$ from the lattice periodicity $\left(d_{z}=1.94 \mathrm{~mm}\right)$ and are used to solve Eq. (12) to compare the theoretical and numerical methods. The theoretical dispersion [Fig. 2(b)] has minima at 148 and $169 \mathrm{GHz}$ - both within $\pm 2 \mathrm{GHz}$ of the frequencies calculated by CST MWS [Fig. 2(a)]. Together, these dispersions predict the existence of possible coupled modes at these frequencies. Although viable coupling mechanisms may be observed in the CST MWS dispersion diagrams, in practice, losses may influence the structure's ability to support a coupled eigenmode [33].

Recent experimental measurements of similar PSL structures show that, at low incident angles, the surface field can manifest up to $10 \mathrm{GHz}$ higher than predicted from the theoretical approximation $\lambda_{s} \cong d_{z}$. The inclusion of the volume field's \pm 1 spatial harmonics may also modify the dispersion in this region, contributing to this disparity. Taking into account the differences in geometry, good agreement between numerical and analytical studies is obtained.

It is observed that $\alpha$ determines the branch separation and influences the overall appearance of the dispersion diagrams [15]. Through extensive study, it is possible to estimate $\alpha$ based on the characteristics and appearance of the structure's dispersion and the frequencies of the coupled eigenmodes. In the CST MWS model, $\alpha$ is varied implicitly by changing the corrugation depth and substrate dimensions of the PSL. Several iterations are carried out in this study and a large number of theoretical and numerical dispersions are calculated to obtain the closest match. The theoretical and numerical simulation data correlate best when Eq. (12) is solved for $\alpha=0.45$. Comparing these methods gives a value of $\alpha$ for the CST MWS planar PSL model. Physical aspects neglected by the CST MWS Eigenmode Solver are the Ohmic and dielectric losses associated with the copper lattice and dielectric substrate.

\section{EXPERIMENTAL MEASUREMENTS}

Figure 3 shows the experimental results for a planar PSLDM with $d_{z}=1.94 \mathrm{~mm}$, mounted on a $0.76-\mathrm{mm}$ copper-backed substrate (Fig. 1) and measured [34] over a range of incident angles $\left(\theta_{i}\right)$ using a Vector Network Analyzer (VNA) at 140-220 GHz [35]. A well-defined resonance, indicative of a coupled-cavity eigenmode, is observed. This resonance is "mode-locked" at a particular frequency, located between its partial volume and surface mode constituents. In Fig. 3, mode locking is observed for $\theta_{i}=50^{\circ}-70^{\circ}$. The inset of Fig. 3 shows uncoupled surface-mode resonances observed in a 1.94-mm-"mesh" PSL structure with no substrate [35]. Measurements of "mesh" PSLs without substrates, used to study the surface field exclusively, show that the surface field frequency shifts down with increasing $\theta_{i}$. It is likely that the surface field frequency being in closer proximity to that of the volume field at higher $\theta_{i}$ better facilitates mode coupling and coupled eigenmode formation. Importantly, this coupled eigenmode dominates over other behavior, with no resonances evident at higher frequencies $(155-220 \mathrm{GHz})$. The observation of this sharp approximately $-40 \mathrm{~dB}$ resonance at a single frequency indicates the structure's potential for use as a perfect absorber and solar cell exploitation. This "mode-locked" coupled eigenmode validates the theoretical and numerical concepts in this paper and demonstrates the principle of mode selection in a large cavity facilitating high-power radiation sources at high frequencies. Such behavior exists only when the PSL is suitably synchronized by the volume mode contained within the substrate. The structure's copper backing facilitates coupling by better synchronizing the individual lattice perturbations and increasing the cavity quality. Without this copper 


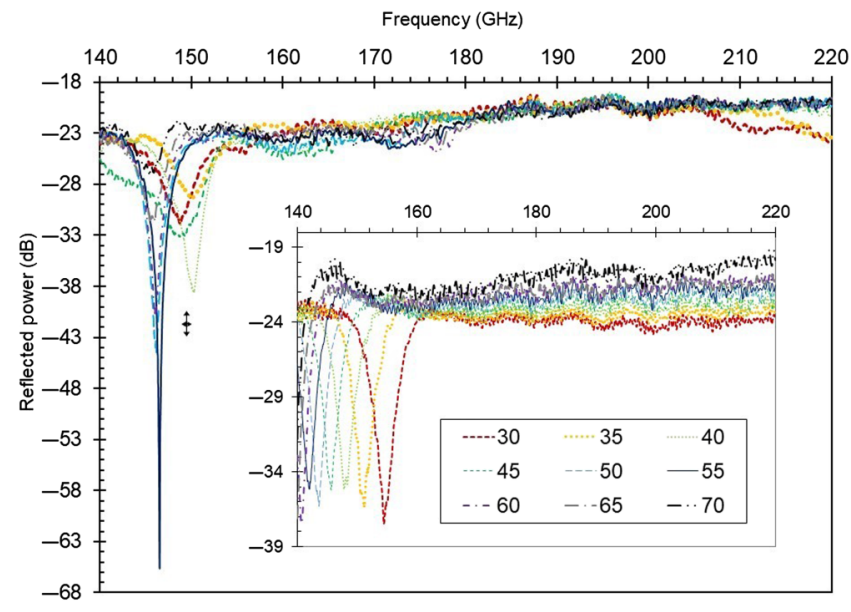

FIG. 3. Coupled eigenmode observed over a range of incident angles from $30^{\circ}$ to $70^{\circ}$ for a $1.94-\mathrm{mm}$ planar PSL-DM etched onto a $0.76-\mathrm{mm}$ copper-backed substrate. The copper backing enhances lattice synchronization and facilitates coupling of volume and surface fields. The inset shows surface-field resonances that shift down in frequency with increasing angle, observed in the case of a 1.94-mm PSL with no substrate [30]. A representative error bar is shown. The magnitude error is $\pm 1 \mathrm{~dB}$ and the frequency error is $\pm 0.1 \mathrm{GHz}$.

backing, volume and surface mode resonances shift down in frequency with increasing $\theta_{i}$.

At low incident angles, there is a larger frequency separation $\Delta$ between the volume and surface modes. Optimal "mode-locking" is, therefore, observed at $\theta_{i}>40^{\circ}$. However, because the dispersions are solved assuming normal incidence, the experimental result is best compared to the theoretical and numerical predictions at the lowest measured angle, $\theta_{i}=30^{\circ}$. The experimental eigenmode of Fig. 3 (located at $149 \mathrm{GHz}$ and measured at $\theta_{i}=30^{\circ}$ ) correlates with both the theoretical and numerical dispersion diagrams that show clear potential for an eigenmode around this frequency. Specifically, the measured resonance shows good agreement with the minimum frequency $(150 \mathrm{GHz})$ of the CST MWS dispersion [Fig. 2(a)] Hence, we conclude that experimental measurements of the $1.94-\mathrm{mm}$ planar PSL, shown in Fig. 3, match the theoretical and numerical predictions when $\alpha \sim 0.45$.

\section{CONCLUSIONS}

The phenomenon of volume and surface mode coupling is demonstrated with good overall agreement between theory, simulations, and experiment. A frequency-locked eigenmode is measured at the $140-220 \mathrm{GHz}$ band for a planar PSLDM structure with a period of $1.94 \mathrm{~mm}$, mounted on a copper-backed substrate. The frequency at which the coupled eigenmode is formed determines the PSL's operating wavelength and is controlled by varying the structure's parameters. Importantly, PSLs are scalable and the theory presented here is applicable over a wide frequency spectrum, from infrared to $\mathrm{THz}$ and beyond, enhancing the broad interest of the work. Following this "proofof-principle," the planar structures can be mapped into cylindrical metallic PSLs compatible with electron beams.

Using the method of fictitious magnetic sources, we explore some of the possible scattering mechanisms and derive a coupling coefficient, $\alpha$. Theoretical and numerical dispersion diagrams successfully predict frequencies at which coupled eigenmodes are possible and give an indication of the strength of the coupling of volume and surface modes. We estimate that $\alpha \sim 0.45$ for the $1.94-\mathrm{mm}$ planar PSL and this method of approximating $\alpha$ will be implemented in future radiation-source experiments. The ability to excite a single high Q mode in a large cavity has relevance and importance for electromagnetic radiation sources based on several media types, that is, free electron radiation sources as well as radiation emission from condensed matter gain media. These results are also applicable for radiation absorption applications such as increased efficiency solar cells and thermal panels.

\section{ACKNOWLEDGMENTS}

The Leverhulme Trust, UK, has provided funding through an International Network Grant No. IN-2015-012. This research has received support from AFOSR under Grants No. FA8655-13-1-2132 and No. FA9550-17-10095 .

[1] J. T. Shen, P. B. Catrysse, and S. Fan, Mechanism for Designing Metallic Metamaterials with a High Index of Refraction, Phys. Rev. Lett. 94, 197401 (2005).

[2] R. A. Shelby, D. R. Smith, and S. Schultz, Experimental verification of a negative index of refraction, Science 292, 77 (2001).

[3] A. Berrier, M. Mulot, M. Swillo, M. Qiu, L. Thylén, A. Talneau, and S. Anand, Negative Refraction at Infrared Wavelengths in a Two-Dimensional Photonic Crystal, Phys. Rev. Lett. 93, 073902 (2004).

[4] H. Shin and S. Fan, All-Angle Negative Refraction for Surface Plasmon Waves Using a Metal-Dielectric-Metal Structure, Phys. Rev. Lett. 96, 073907 (2006).

[5] A. N. Grigorenko, A. K. Geim, H. F. Gleeson, Y. Zhang, A. A. Firsov, I. Y. Khrushchev, and J. Petrovic, Nanofabricated media with negative permeability at visible frequencies, Nature 438, 335 (2005).

[6] D. R. Smith, W. J. Padilla, D. C. Vier, S. C. Nemat-Nasser, and S. Schultz, Composite Medium with Simultaneously Negative Permeability and Permittivity, Phys. Rev. Lett. 84, 4184 (2000).

[7] S. Zhang, W. Fan, B. K. Minhas, A. Frauenglass, K. J. Malloy, and S. R. J. Brueck, Midinfrared Resonant Magnetic Nanostructures Exhibiting a Negative Permeability, Phys. Rev. Lett. 94, 037402 (2005). 
[8] G. Dolling, C. Enkrich, M. Wegener, C. M. Soukoulis, and $\mathrm{S}$. Linden, Simultaneous negative phase and group velocity of light in a metamaterial, Science 312, 892 (2006).

[9] C. Enkrich, M. Wegener, S. Linden, S. Burger, L. Zschiedrich, F. Schmidt, J. F. Zhou, Th. Koschny, and C. M. Soukoulis, Magnetic Metamaterials at Telecommunication and Visible Frequencies, Phys. Rev. Lett. 95, 203901 (2005).

[10] S. Linden, C. Enkrich, M. Wegener, J. Zhou, Th. Koschny, and C. M. Soukoulis, Magnetic response of metamaterials at 100 terahertz, Science 306, 1351 (2004).

[11] T. J. Yen, W. J. Padilla, N. Fang, D. C. Vier, D. R. Smith, J. B. Pendry, D. N. Basov, and X. Zhang, Terahertz magnetic response from artificial materials, Science 303, 1494 (2004).

[12] J. B. Pendry, Negative Refraction Makes a Perfect Lens, Phys. Rev. Lett. 85, 3966 (2000).

[13] D. Schurig, J. J. Mock, B. J. Justice, S. A. Cummer, J. B. Pendry, A. F. Starr, and D. R. Smith, Metamaterial electromagnetic cloak at microwave frequencies, Science 314, 977 (2006).

[14] I. V. Konoplev, A. J. MacLachlan, C. W. Robertson, A. W. Cross, and A. D. R. Phelps, Cylindrical periodic surface lattice as a metadielectric: Concept of a surface-field Cherenkov source of coherent radiation, Phys. Rev. A 84, 013826 (2011).

[15] I. V. Konoplev, A. J. MacLachlan, C. W. Robertson, A. W. Cross, and A. D. R. Phelps, Cylindrical, periodic surface lattice-Theory, dispersion analysis, and experiment, Appl. Phys. Lett. 101, 121111 (2012).

[16] N. S. Ginzburg, A. M. Malkin, A. S. Sergeev, and V. Yu. Zaslavsky, Oversized co-axial and cylindrical surface-wave oscillators with two-dimensional periodical grating (quasioptical model), J. Appl. Phys. 113, 104504 (2013).

[17] N. S. Ginzburg, E. V. Ilyakov, I. S. Kulagin, A. M. Malkin, N. Yu. Peskov, A. S. Sergeev, and V. Yu. Zaslavsky, Theoretical and experimental studies of relativistic oversized Ka-band surface-wave oscillator based on 2D periodical corrugated structure, Phys. Rev. Accel. Beams 21, 080701 (2018).

[18] D. M. French and D. Shiffler, High power microwave source with a three dimensional printed metamaterial slow-wave structure, Rev. Sci. Instrum. 87, 053308 (2016).

[19] S. Liu, P. Zhang, W. Liu, S. Gong, R. Zhong, Y. Zhang, and $\mathrm{M}$. Hu, Surface Polariton Cherenkov Light Radiation Source, Phys. Rev. Lett. 109, 153902 (2012).

[20] N. I. Landy, S. Sajuyigbe, J. J. Mock, D. R. Smith, and W. J. Padilla, Perfect Metamaterial Absorber, Phys. Rev. Lett. 100, 207402 (2008).

[21] E. Abraham, A. Younus, A. El. Fatimy, J. C. Delagnes, E. Nguéma, and P. Mounaix, Broadband terahertz imaging of documents written with lead pencils, Opt. Commun. 282, 3104 (2009).
[22] G. L. Carr, M. C. Martin, W. R. McKinney, K. Jordan, G. R. Neil, and G. P. Williams, High-power terahertz radiation from relativistic electrons, Nature 420, 153 (2002).

[23] C. Luo, S. G. Johnson, J. D. Joannopoulos, and J. B. Pendry, Subwavelength imaging in photonic crystals, Phys. Rev. B 68, 045115 (2003).

[24] E. Lier, D. H. Werner, C. P. Scarborough, Q. Wu, and J. A. Bossard, An octave-bandwidth negligible-loss radiofrequency metamaterial, Nat. Mater. 10, 216 (2011).

[25] F. Benabid, J. C. Knight, G. Antonopoulos, and P. St. J. Russell, Stimulated Raman scattering in hydrogenfilled hollow-core photonic crystal fiber, Science 298, 399 (2002).

[26] Yu. A. Grishin, M. R. Fuchs, A. Schnegg, A. A. Dubinskii, B. S. Dumesh, F. S. Rusin, V. L. Bratman, and K. Möbius, Pulsed Orotron - A new microwave source for submillimeter pulse high-field electron paramagnetic resonance spectroscopy, Rev. Sci. Instrum. 75, 2926 (2004).

[27] G. Doucas, V. Blackmore, B. Ottewell, C. Perry, P. G. Huggard, E. Castro-Camus, M. B. Johnston, J. Lloyd Hughes, M. F. Kimmitt, B. Redlich, and A. van der Mee, Longitudinal electron bunch profile diagnostics at $45 \mathrm{MeV}$ using coherent Smith-Purcell radiation, Phys. Rev. ST Accel. Beams 9, 092801 (2006).

[28] A. G. York, H. M. Milchberg, J. P. Palastro, and T. M. Antonsen, Direct Acceleration of Electrons in a Corrugated Plasma Waveguide, Phys. Rev. Lett. 100, 195001 (2008).

[29] M. van Lare, F. Lenzmann, M. A. Verschuuren, and A. Polman, Mode coupling by plasmonic surface scatterers in thin-film silicon solar cells, Appl. Phys. Lett. 101, 221110 (2012).

[30] W. S. Lee, E. Ott, and T. M. Antonsen, Large Coupled Oscillator Systems with Heterogeneous Interaction Delays, Phys. Rev. Lett. 103, 044101 (2009).

[31] G. Burt, S. V. Samsonov, K. Ronald, G. G. Denisov, A. R. Young, V. L. Bratman, A. D. R. Phelps, A. W. Cross, I. V. Konoplev, W. He, J. Thomson, and C. G. Whyte, Dispersion of helically corrugated waveguides: Analytical, numerical, and experimental study, Phys. Rev. E 70, 046402 (2004).

[32] The Computer Simulation Technology (CST) Microwave Studio (MWS) Eigenmode Solver is described at https:// www.cst.com/products/cstmws/solvers/eigenmodesolver

[33] I. V. Konoplev, A. D. R. Phelps, A. W. Cross, and K. Ronald, Experimental studies of the influence of distributed power losses on the transparency of two-dimensional surface photonic band-gap structures, Phys. Rev. E 68, 066613 (2003).

[34] A. J. MacLachlan, C. W. Robertson, A. W. Cross, and A. D. R. Phelps, Volume and surface mode coupling experiments in periodic surface structures for use in $\mathrm{mm}-\mathrm{THz}$ high power radiation sources, AIP Adv. 8, 105115 (2018).

[35] Data underpinning this publication are available from the University of Strathclyde KnowledgeBase at https://doi.org/ 10.15129/41345641-c83f-4af5-a1 14-cb015bc954fd 